\title{
OBituARIOS
}

\section{Prof. Nuria Cortada de Kohan}

El 31 de marzo falleció en Buenos Aires la Profesora Nuria Cortada después de una fructífera vida de 91 años. Nuria fue la Maestra de varias generaciones de psicólogos, sociólogos y educadores que dieron sus primeros pasos en el aprendizaje de la estadística y la investigación de su mano.

Nuria había nacido el 5 de noviembre de 1921 en Mendoza. Después de hacer sus estudios primarios en Barcelona, regresó a Mendoza donde terminó su formación secundaria y universitaria. Allí comenzó también su trabajo en investigación con el Dr. Horacio Rimoldi al que fue presentada por el Dr. Emilio Mira y López. En el Instituto Nacional de Psicología Experimental de la Universidad Nacional de Cuyo, dirigido por el Dr. Rimoldi, realizó estudios de avanzada para la época, participando entre otras labores psicométricas pioneras en Argentina, en la primera tipificación nacional del Test de Raven en 1943.

En 1946 ganó una beca del Institute for International Education para estudiar en la Ohio State University, donde obtuvo en 1947 el Master of Arts en Psicología.

En 1952 ganó también la beca denominada Etudiante Patronée concedida por el Gobierno de Francia para estudiar Psicología en La Sorbonne de París, bajo la dirección del Profesor Dr. Pierre Pichot.

En 1971 fue invitada especial del Dr. Lee Cronbach, para una estadía en la Social Research Foundation de los Estados Unidos.

Si bien ya había tenido varias cátedras en la Facultad de Filosofía y Letras de la Universidad de Buenos Aires (UBA), recién en 1959 es nombrada Profesora Titular de Metodología Estadística con dedicación exclusiva. Desde ese cargo, tiene un gran protagonismo en el crecimiento de la Carrera de Psicología, recién creada, y trabaja incansablemente por dar a conocer y hacer crecer una psicología enfocada en la psicometría y en los principios de la ciencia en general, bastante distinta a la predominante en esa época.

En 1964 y luego de ocupar varios cargos en el Departamento de Orientación Vocacional de la UBA es nombrada Directora del mismo. En 1968 se crea la Cátedra de Metodología de la Investigación Psicológica y Nuria fue designada Profesora Titular.

En 1969 el Dr. Rimoldi es repatriado por el Dr. Houssay y se crea en Buenos Aires el Centro Interdisciplinario de Investigaciones en Psicología Matemática y Experimental (CIIPME) en cuyo proceso de organización Nuria tuvo un protagonismo fundamental.

Fue miembro del Comité Editorial y una gran colaboradora de INTERDISCIPLINARIA.

Luego vendrán tiempos difíciles en los que se aleja de la Facultad de Psicología de la UBA y obtiene un contrato como Investigador Principal del CONICET en el CIIPME y entra un poco más adelante en la Universidad del Salvador donde permanecería hasta su muerte.

En 1996 es nombrada Profesora Honoraria de la UBA y en 2011 recibe un importante reconocimiento por su trayectoria académica, en el marco del $190^{\circ}$ Aniversario de la Fundación de la UBA. También ese año es nombrada Profesora Emérita por la Facultad de Psicología y Psicopedagogía de la Universidad del Salvador (USAL).

Más allá de todos sus importantísimos antecedentes como académica, Nuria ha sido un gran ser humano, de una generosidad poco común con todos sus alumnos y discípulos, además de ser una persona con una gran humildad y paciencia para enseñar, siempre dispuesta a compartir sus conocimientos o su excelente biblioteca. 
Su actitud optimista hacia la vida, el ir para adelante superando los problemas, su risa pronta y contagiosa, fueron algunas de sus muchas virtudes.

Hasta poco antes de morir mostró siempre un renovado entusiasmo, permanente búsqueda de nuevos conocimientos y alegría frente a la vida. Fue además una invalorable amiga de todos los que estuvimos cerca de ella.

Adiós querida Nuria. Siempre estará en el corazón de los muchos que la queremos y respetamos.

María CRISTINA Richaud

\section{Autobiografía de Nuria Cortada de KoHan*}

Nací en Mendoza el 5 de noviembre de 1921 y viví allí hasta los 4 años, cuando mis padres, oriundos de Catalunya, deciden volver a España.

Mi educación primaria fue como anárquica, ya que asistí durante muy poco tiempo a la escuela; sin embargo, parecería no haber sido tal pues mi padre, médico, catalán y muy republicano, muy culto y muy emprendedor, fue un cuidadoso organizador de mi carrera académica. También mi madre, brillante maestra y sus dos hermanos, 10 años mayores, me dieron la formación básica. La anarquía a la que me refiero expresa el sentimiento que experimenté de niña debido a las idas y venidas de la familia entre Barcelona y Mendoza, según los vaivenes políticos de Europa, por lo que recibí la formación fundamentalmente en la familia. Además estudié varios idiomas con institutrices. Viví en Barcelona desde los 4 a los 15 años y cuando estalla la Guerra Civil Española, en 1936, mi familia emigra a Argentina. Esta formación, variada y ecléctica, incluyó un aspecto importante como instrumento de mi futura actividad: el aprendizaje y el gusto por la matemática, los idiomas, la música, las personas y los viajes.

Lo que más recuerdo es nuestro perro, un precioso setter que merece estar en el paraíso perruno, pues aguantaba mis caricias y ejercicios como un santo. Esto debe haber fijado en mí el gran cariño que siempre he sentido por los perros y hace que usualmente tenga alguno cerca de mí. También vislumbro el color lila de una enorme glicina que perfumaba con sus flores el patio y bajo la cual jugaba con las cajitas vacías de inyectables que me daba mi padre al salir de su consultorio y que eran mis tesoros.

A los 4 años mis padres volvieron a Europa y en el trasatlántico, mi madre en 15 días, me enseñó a leer. Llegamos a Barcelona y allí descubrí que tenía un hermano y una hermana, 10 y 9 años mayores que yo y que funcionaron como otro par de padres con el mismo cariño y autoridad que los propios. Ellos se habían adelantado un año al viaje a Barcelona con mi abuela, para iniciar sus estudios secundarios.

Mi educación primaria fue muy anárquica. Comencé a ir a la escuela a los 6 años; pero cada 2 ó 3 días volvía a mi casa con anginas o alguna enfermedad infantil y mi padre a la cuarta o quinta repetición, decidió que sería mejor que aprendiera en casa con mi madre y mis hermanos. Mi madre era

* Los editores agradecen a su hija Ana Kohan Cortada, quien gentilmente facilitó y autorizó la publicación de este documento. 
maestra y mi hermano que entonces estaba terminando el bachillerato, tenía un don especial para enseñarme Matemática. Debo decir que mis padres tenían ideas muy abiertas: nunca nos impusieron nada, ni tampoco nos prohibieron nada. Siempre leí cuanto libro caía en mis manos y mi casa estaba llena de libros. Para nuestros padres solo dos valores eran los fundamentales, la educación y la honestidad. No nos inculcaron ideas religiosas ni políticas de ninguna clase; pero siempre propiciaron toda actividad que tuviera que ver con el arte, la cultura, la salud, la historia, la ciencia o las letras. Muy pronto, a los 7 años, me pusieron una profesora de francés y a los 8 ó 9 años empecé a aprender a tocar el piano y a estudiar inglés y alemán. Debo decir que los idiomas siempre me resultaron fáciles pues he sido siempre bilingüe hablando y escribiendo desde muy pequeña, en castellano y en catalán. A los 10 años di examen de ingreso para comenzar el bachillerato y entonces sí, entre los 11 y 14 años cursé 4 años de bachillerato regularmente.

Fueron años muy felices. Con mi familia pasaba parte del verano en una playa de la Costa Brava sobre el azul Mediterráneo y parte en la casa en que mi madre había nacido, en un pueblito del interior, cerca de Barcelona, en donde se cultivan viñedos. Durante el invierno concurríamos a cuanto espectáculo había: allí pude escuchar a Pablo Casals, a Rubinstein, a Stern, a Horovitz y a tantos otros. En esta época empecé a leer Alicia en el País de las Maravillas, los Cuentos de Calleja, los Cuentos de Andersen, las novelitas de Salgari, las aventuras de Julio Verne, Mark Twain, Edgar Alan Poe, Dickens, etc. Luego seguí leyendo casi todos los clásicos españoles y franceses, pues en esta época comenzó mi costumbre que no he dejado nunca, de leer un ratito en la cama antes de dormir. Creo que es importante hacer notar aquí que mi padre era una persona muy inteligente y que pensaba que las chicas tenían tanto derecho a estudiar y a formarse intelectualmente como los jóvenes; además era muy culto, leía en varios idiomas y lo que más le preocupaba sobre los hijos era que tuvieran una buena formación intelectual. Mi hermano a los 21 años se graduó de médico y mi hermana también estudiaba y mi casa cuando yo era muy pequeña, estaba siempre llena de jóvenes estudiantes y todos jugaban y se reían conmigo. Los domingos por la tarde íbamos al cine con todos los primos indefectiblemente y también recuerdo estos años por las grandes amistades con compañeras de curso y las interminables y románticas conversaciones sobre si era mejor actor o más atrayente, Glark Gable o Gary Cooper! Pero, ¡ay! parece que nada bueno perdura y en un fatídico julio de 1936 empezó la Guerra Civil Española. Mis padres decidieron volver a la Argentina y un 5 de noviembre, el día que cumplía 15 años, llegamos a Buenos Aires. Teníamos poco más que lo puesto, pues no habíamos podido traer casi nada de la casa. Alli quedaron muebles, ropa, piano, libros y diccionarios en manos de mi pobre abuela a quien no pude ver más. Fue un desastre general y familiar que me hizo crecer de golpe.

Seguramente se habrá advertido que fui bastante mimada por la suerte en mi infancia, y aunque era muy leida como algunos dirían, también era bastante inmadura. Viví en esta época un año en Buenos Aires haciendo trámites para lograr la equivalencia que el Ministerio de Educación hizo de mis estudios secundarios. Lo que más me gustó de Buenos Aires fueron los enormes árboles de las plazas, los inmensos gomeros de la Plaza San Martín y Lavalle y también el delicioso color de los jacarandaes en flor. Eso no lo teníamos en Barcelona, y me hacía sentir que estábamos realmente en la América de los libros! Al terminar los trámites de equivalencia, tras algunas indecisiones familiares que no vienen al caso, mis padres volvieron conmigo a Mendoza, en donde algún tiempo después terminé el bachillerato dando casi todas las materias como alumna libre en el Colegio Nacional Agustín Alvarez. Creo que esto fue, a pesar de todo el esfuerzo que me costó, un gran aprendizaje pues al tener que dar tantas materias como libre aprendí mucho a estudiar y a enfrentar las situaciones de examen. 
Y llegó el momento de decidir sobre mis futuros estudios. Yo seguía leyendo mucho. En esta época me interesaba sobre todo la literatura rusa y el enfoque psicológico de los angustiados personajes de Dovstoyewski y de Tolstoi me apasionaba más que nada y comencé a pensar que eso era lo que me gustaría saber a fondo. Mi padre conversaba mucho conmigo. Mis hermanos se habían casado y vivían lejos; así que en este momento yo era su mayor preocupación y se daba cuenta de que era importante que cualquier carrera que eligiera, debía hacerla con gusto. Entonces en un viaje a Buenos Aires, lo visitamos al Dr. Emilio Mira y López que mi padre conocía mucho de Barcelona y le pedimos que me orientara vocacionalmente. Fui un día sola; el Dr. Mira habló largamente conmigo de muchas cosas, por cierto no me hizo ningún test, y cuando mi padre me vino a buscar le dijo: " $\mathrm{E}$ Esta chica puede seguir cualquier carrera; en cualquier cosa va a tener éxito!" Yo me reía, lo que quería es que me dijera qué carrera seguir, si Medicina o Filosofía. Pero Mira dijo: "Si le interesa Psicología va a tener que irse al extranjero, acá la carrera no existe." $Y$ en ese momento se quedó callado, pensando, y de pronto dijo: "iAh! Ahora recuerdo que Houssay me dijo el otro día, que pronto llegaría a Mendoza un profesor de Psicología contratado por la Universidad Nacional de Cuyo, que viene de Inglaterra y está muy preparado en Psicología. Pónganse en contacto con él, se llama Horacio Rimoldi".

Y así es como decidí estudiar Filosofía y Letras en Mendoza. Mi paso por la Universidad de Cuyo fue breve, pero muy agradable. La universidad, que hacía apenas un año se había creado, tenía un rector, el Dr. Edmundo Correas, que con criterio muy amplio, hizo nombrar a profesores jóvenes, todos muy prometedores. Aparte del Dr. Rimoldi con quien me formé aprendiendo en su Instituto de Psicología Experimental, recuerdo bien a otros profesores como el Dr. Pro, el Dr. Juan Corominas, a Julio Cortázar, a Manlio Lugaresi, a Roberto Salmón, al Dr. Mavridis, al Dr. García de Onrubia, al Dr. Cruz, a Horacio Schindler, etc. También recuerdo con afecto, aunque a muchos de ellos no los he vuelto a ver, a mis compañeros, los hermanos Roig, a Zamorano, al malogrado Mauricio López, a las hermanas Quiroga, a Susana Velasco, a Hilda Calderón, a Irma Suárez, etc. etc. Terminé la carrera de Filosofía en 4 años (pues di un año libre), pero creo que fueron muy formativos. Sobre todo, la posibilidad sin par, de trabajar al lado del Dr. Rimoldi plasmó definitivamente mi vocación. A él debo gran parte de lo que soy y por esto le estaré siempre agradecida. Con otros ayudantes del Instituto como Vevette Metraux, Raquel San Martín, Susana Velazco y Lidia Bührer, formábamos un equipo lleno de entusiasmo y entre risas y tropiezos aprendimos del Dr. Rimoldi no solo los primeros pasos de la investigación científica, sino la importancia del esfuerzo y el trabajo sostenido y el saber seguir siempre adelante y no ceder a pesar de las dificultades y frustraciones. El Dr. Rimoldi había organizado el Instituto de Psicología Experimental en la Universidad de Cuyo con gran entusiasmo. Con él tuve la oportunidad de aprender trabajando las técnicas estadísticas descriptivas y de correlación. Allí se hizo la primera estandarización del Test de Raven en el país, en 1943. Creo que este ha sido un trabajo bastante importante por ser la primera vez que en la Argentina se estandarizaba un instrumento de medición psicológica. Tomamos el test en forma individual a más de 1.400 niños de las escuelas de Mendoza y realizamos todo el trabajo estadístico necesario para tener un verdadero baremo con los valores descriptivos, la transformación en percentiles, la comparación con los valores hallados en Inglaterra, etc. En el Instituto también se encararon otros problemas, como los de adecuación al trabajo y un estudio comparativo de algunas funciones psicomotoras entre débiles físicos y normales. Todos estos trabajos aparecieron en una serie de publicaciones del Instituto entre 1943 y 1945.

Al terminar la carrera de Filosofía y Letras, en 1945 me presenté a concurso para una cátedra de Psicología Infantil en la Escuela Normal Pringles de San Luis. Aquella fue mi primera experiencia docente. 
Recuerdo que sentía bastante miedo de enfrentar a los alumnos, pero el Profesor Plácido Horas siempre tan comprensivo, me animó mucho y así salí del paso. Desde entonces siempre mantuve con el Profesor Horas una profunda y sincera amistad.

Enseguida gané mi beca del Institute of International Education para estudiar en los Estados Unidos. Ahora viajar es cosa de todos los días, pero entonces todavía se viajaba en barco y para una joven sola era toda una hazaña. Recuerdo que el viaje me pareció interminable pues era un buque de carga cuya única ventaja fue la de entrar en muchos puertos y estar allí 2 ó 3 días, lo que me permitió conocer Río de Janeiro, Santos, Curaçao, La Habana, Norfolk y finalmente Nueva York. Estuve 5 días en Nueva York y a principios de septiembre de 1946 llegué a la Universidad del Estado de Ohio en Columbia para estudiar Psicología. En esta época The Ohio State University era un centro muy importante para la Psicología Clínica. Allí había una serie de seguidores del Dr. Carl Rogers, entre ellos, el Dr. Victor Raimy, una persona extraordinaria que fue mi adviser. Además estaban el Dr. George Kelly, conocido por su teoría de los personal constructs, el Dr. Julian Rotter, que enseñaba técnicas proyectivas, el Dr. Renshaw que era un maestro muy reconocido en Psicología de la Gestalt, el Dr. Toops que era el terror de los alumnos en Estadística, el Dr. John Horrocks especialista en Psicología Infantil, etc. Tuve mucha suerte en estudiar con todos estos profesores que me hicieron conocer la Psicología Clínica y me trataron no solo muy bien, sino que casi podría decirse que me mimaron, pues les parecía raro que hubiera ido de tan lejos -la Argentina- a estudiar en Ohio. En esos años el Dr. Rimoldi también estaba en los Estados Unidos, en la Universidad de Chicago y solía invitarme durante las breves vacaciones a pasar unos días en su casa. Así fue como pude conocer muy de cerca al Dr. Thusrtone y también a algunos de sus discípulos como el español Mariano Yela, con quien siempre mantuve una buena amistad. Estudié en los Estados Unidos casi 3 años: obtuve el Master of Arts en Psicología Clínica y entonces decidí volver a Buenos Aires donde estaban mis padres. Podría haberme quedado en los Estados Unidos... nunca sabré si mi decisión de volver a la Argentina fue acertada o no...

Aquellos años en Buenos Aires fueron difíciles. Conocía a poca gente, tenía que trabajar y empecé a moverme. "¿Qué es un psicólogo?" me preguntaba mucha gente. En este momento pocos sabían que era eso. He sido la primera persona en mi país con título de Psicóloga. Entré a trabajar en el Ministerio de Educación en un Centro de Orientación Vocacional y Educativa que estaba en el Instituto Bernasconi y luego en Sanidad Escolar. Fue en esta época que aproveché el tiempo, tipificando el Test de Raven para la ciudad de Buenos Aires. Un día, casualmente, me encontré en la calle con el Dr. Felipe García de Onrubia. El me conocía de Mendoza y fue muy amable conmigo haciéndome entrar en la Universidad de Buenos Aires como Jefe de Seminario en la materia Psicología II que él dictaba en la carrera de Filosofía. Allí di varios seminarios sobre Estadística Aplicada y teoría de los tests. En este momento, entre 1950 y 1955, ya se estaba despertando en muchas personas el interés y la necesidad de que se creara la carrera de Psicología: existía un centro muy bueno en el Hospital de Clínicas, el de Psiquiatría Infantil de la Dra. Telma Reca con quien yo me había puesto en contacto y a donde concurría algunos días de la semana para aplicar el Test de Rorschach a los niños que ella me indicaba. También estaba en contacto a menudo con la Dra. Carolina Tobar García y su grupo de visitadoras sociales, algunas de las cuales trabajaban conmigo en el Centro de Orientación Vocacional y Educativa.

En 1952 gané la beca francesa para estudiar en París. Esto fue para mi muy positivo. Trabajé alrededor de 9 meses en el Hospital Sainte Anne bajo el asesoramiento del Dr. Pierre Pichot haciendo sobre todo Psicometría. Tuve oportunidad de conocer a Faverge y a Zazzo, y de seguir un curso con ellos: pero sobre todo esta beca me hizo tomar conciencia de que mi preparación era muy superior en Psico- 
logía, a la de muchos jóvenes franceses. En este viaje tuve oportunidad de conocer Francia, Inglaterra, Italia y finalmente España en donde tenía a mis tíos. España en pleno franquismo me pareció muy triste, opresiva, sobre todo al recordar los días luminosos de la República, que siendo chica había vivido. Aunque también allí estuve en contacto con el Dr. Mariano Yela que había conocido en Chicago y me hizo ver los esfuerzos de algunas personas como él mismo y el Profesor Germain por iniciar una Psicología científica en España.

En 1954 el Prof. Oñativia y el Prof. Moreno de Tucumán tuvieron la oportuna idea de organizar el Primer Congreso Argentino de Psicología. Allí nos encontramos muchos especialistas, médicos, profesores, psicoanalistas, etc. que teníamos la idea de que era necesario crear la Carrera de Psicología y tuvimos algunas primeras conversaciones. En Buenos Aires el Dr. Gino Germani estaba interesado en la creación de la Carrera de Sociología. El fue quien me llamó a una primera reunión, pues sabía de mi formación en Estadística Aplicada y me pidió que colaborara con él y con la Dra. Telma Reca, el Prof. Jaime Berstein y el Dr. Marcos Victoria, para la elaboración de los primeros planes de estudio de Psicología en la Universidad de Buenos Aires. Era la única psicóloga del grupo, dado que todos eran médicos, profesores en Filosofía o en Ciencias de la Educación. Pronto se nos unieron otras personas interesadas, y en 1957 al crear el Dr. Rizieri Frondizi la Carrera de Psicología ya empecé a dictar Metodología Estadística para los alumnos de Psicología y Sociología, primero en forma interina y en 1959 gané por concurso la cátedra que tuve hasta 1984. Estos primeros años fueron la época de oro de las carreras de Psicología y Sociología, mientras fueron rectores de la Universidad de Buenos Aires sucesivamente, el Dr. Risieri Frondizi, el Dr. Olivera y el Ing. Fernández Long. La Carrera de Psicología se inició con gran entusiasmo, tanto por parte de los primeros alumnos como por parte de los profesores. En realidad nos formábamos mutuamente pues nunca se aprende tanto algo como cuando hay que enseñarlo. En mi materia tuve la suerte de contar con algunos jefes de trabajos prácticos y adjuntos muy preparados como el Ing. José Carro, el Ing. Cavallini y la Prof. Malvina Segre y al mismo tiempo al hacerse más numeroso el estudiantado formamos a un gran grupo de ayudantes de todos los cuales me siento muy orgullosa pues actualmente todos ellos son profesores en diversas universidades y siguen trabajando seriamente en investigación como Alfredo López Alonso, Alejandro Doublier, Nélida Rodríguez Feijóo, Cristina Richaud, Dorina Stefani, Miko Mandilovich, Alicia Bertoni, Marta Schufer, Marta Locatelli, Alicia Casullo y otros cuyos nombres he olvidado.

Fue en esta época cuando escribí con Carro mi primer libro, Estadística Aplicada que tuvo gran éxito y del que Eudeba hiciera muchas reediciones. Hay que pensar que enseñar Estadística en esos años era bastante complicado. En primer lugar porque no contábamos como ahora con programas estadísticos. No teníamos computadoras ni fotocopiadoras, ni siquiera las usuales calculadoras de bolsillo. Sin embargo, un verano Malvina y yo nos pasamos 3 meses haciendo los cálculos para un análisis factorial que nos pidió Germani para sus investigaciones sobre el NES (Nivel Económico Social). Creo que este ha sido el primer análisis factorial que se ha realizado en nuestro país. A pesar de la precariedad de los recursos y teniendo en cuenta que la Estadística no estimula precisamente 'los amores fáciles', en mi cátedra se comenzó a formar en investigación un grupo de ayudantes de quienes guardo los mejores recuerdos y sin cuya ayuda y dedicación habría sido muy difícil llevar adelante el trabajo. En fin, no sé si mis alumnos aprendieron mucha Estadística conmigo; pero si sé que yo aprendí mucho de ellos, por lo que siempre recuerdo con nostalgia los primeros años de la creación de la Carrera de Psicología como uno de los períodos más productivos y agradables de mi vida. Todo esto, junto al hecho de tener que tratar de compensar con nuestro esfuerzo la actitud negativa hacia los números que sue- 
len tener quienes están en una Facultad de Filosofía, no hacía nada fácil la enseñanza de la Estadística. A veces me sentía algo deprimida y pensaba que yo sabía poca matemática para enseñar la materia. Un día fui a verlo al Dr. Luis Santaló, el gran matemático, nacido en un pueblito cerca de Gerona, como mi padre, y le expuse mi problema. "Nuria -me dijo - quédate tranquila. La Estadística es una ciencia experimental y aplicada y tú sabes muy bien las aplicaciones a la Psicología. Explica esto que sabes y deja que los matemáticos resuelvan los problemas teóricos." Esto me tranquilizó un poco y seguí adelante.

Alrededor de 1958 fui nombrada Jefe del Departamento de Orientación Vocacional (DOV) de la Universidad de Buenos Aires cuyo primer director fue el Profesor Jaime Berstein, quien estuvo solo un año, luego un tiempo muy corto estuvo el Prof. Nicolás Tavella y finalmente me nombraron Directora y estuve más de 6 años. Si bien la Orientación Vocacional clásica establecía que cada cual debe ocuparse de aquello para lo que está más dotado, nosotros pensábamos que aunque esto es cierto en términos generales, el joven debe orientarse para que sepa mantener la flexibilidad de pensamiento para el constante ajuste al cambio que le exige el mundo moderno y postmoderno. Organizamos el DOV para que siempre se tuvieran en cuenta en la orientación vocacional tres enfoques: el psicológico dirigido al bienestar personal, el educativo, promoviendo el ideal de la educación continua y el socioeconómico para que el hombre colabore con el progreso y desarrollo y se integre al mundo social y del trabajo. En nuestro departamento se subrayó especialmente el aspecto dinámico del proceso de desarrollo vocacional frente a los conceptos clásicos de la elección puntual y estática. Se rechazó la noción del mero ajuste entre persona y trabajo y se valoró en cambio una perspectiva con el conjunto de elecciones que el sujeto realiza a lo largo de la vida, encadenadas unas a otras configurando una 'carrera académica'.

La misma división del trabajo de la primera experiencia del DOV de la UBA acentuó la importancia que dimos a la información, la exploración y la documentación, creando la ya famosa Guía del Estudiante. También se pensó en la evaluación y medición de los constructos con la elaboración de muchos instrumentos propios y otros que fueron estandarizados, y a la orientación psicológica con el enfoque clínico de las entrevistas individuales y grupales. Con esta organización atendíamos a los aspectos cognitivos y a los motivacionales que conforman el modelo de carrera que cada joven es capaz de elegir, a medida que se encamina hacia su maduración y concreta sus imágenes sobre las representaciones sociales de la vida profesional. Siempre tuvimos en cuenta que lo más importante es hacer comprender a los adolescentes que los factores de satisfacción profesional están estrechamente ligados a la libertad y autonomía en la organización y la realización del trabajo, así como en el sentimiento de ser responsables ellos mismos de estos. El Departamento de Orientación Vocacional fue concebido desde el primer momento, no solo como un centro de la Universidad donde podían concurrir los propios alumnos cuando tenían problemas de elección de carrera, sino también como un servicio a la comunidad para jóvenes de todas las escuelas de la Capital que necesitaran orientarse para elegir estudios, y además como un centro de formación e investigación en Orientación Vocacional que se consideraba una especialización muy importante de los psicólogos. Creo que es conveniente que nombre a algunos de los múltiples jóvenes que se formaron allí, entre ellos la actual Directora de la Dirección de Orientación al Estudiante de nuestra Universidad de Buenos Aires, Diana Aisenson. Además entre lo que ahora puedo recordar, pues han pasado muchos años, estaban: Miguelina Guirao, Carlos Cuidet, Ederville Cagnone, Irene Orlando, Nora Sturm, Adela Leivovitch de Duarte, Marta Brea, Rodolfo Bohoslavsky, Sara Slapak, María Martina Casullo, Raquel Lutsky, Clelia Ca, Graciela Canesa, Julia García, Federico Kaufman, Angela Canavesi, Lucy Wolf, Edith Adamosky, Celia Jaes, Nestor Yoguel, Ricardo Sheffick, Liliana Misrahi, Sarita Leibeshutz, Elvira Nicolini, etc. En el DOV de la Universidad de Buenos Aires tuvimos horas más felices y menos felices, 
tuvimos mucho diálogo y también controversia; pero no me cabe duda alguna de que todos los que participamos en aquella época lo recordamos con gran afecto y un dejo de nostalgia. En esta época tuve oportunidad de realizar una gran cantidad de tipificaciones de tests para orientación vocacional como el DAT, el Test de las Aptitudes Primarias de Thurstone y otros. Cuando dejé el DOV, escribí un libro sobre el proceso de orientación, El Profesor y la Orientación Vocacional que fue publicado en 1977 por la Editorial Trillas con un prólogo del Dr. Wayne Holtzman y del que se han hecho múltiples reediciones. Estos fueron años muy productivos.

En 1956 me casé y debo decir que fue gracias a la enorme comprensión y compañerismo de mi esposo que pude desarrollar tanta actividad. Durante muchos años tuve la Cátedra de Metodología Estadística, dirigía el Departamento de Orientación Vocacional, formé parte de innumerables jurados, concurrí a múltiples congresos y jornadas, di algunos cursos de Estadística en La Plata y en Salta y todavía me quedó tiempo para traducir del inglés más de una docena de libros, algunos de ellos muy importantes como La Psicología Experimental de Woodwoth y Schlosberg, para EUDEBA, La Estadística de Yamane y La Naturaleza de la Inteligencia de Guilford para la Editorial Paidós. En 1961, con mi esposo decidimos probar suerte en los Estados Unidos. Allí viajamos con nuestra hijita de 6 meses hasta que cumplió 2 años. Era un año difícil para los Estados Unidos. Para mi esposo, que era Ingeniero Agrónomo fue muy difícil. Pero yo encontré trabajo como Senior Clinical Psychologist en el Saint Lawrence State Hospital, un hospital psiquiátrico al norte del Estado de Nueva York sobre el río Saint Lawrence, a 50 millas de Otawa. En este hospital por primera vez trabajé con toda la responsabilidad de un psicólogo clínico, realizando muchos psicodiagnósticos para los psiquiatras, organizando grupos operativos con los pacientes, la mayoría de los cuales eran dipsómanos y estuve en contacto directo con la experiencia psiquiátrica de aquel momento, que por cierto era bastante traumática y deprimente. Pasamos allí un año; pero se nos terminaban las licencias de nuestros trabajos en Buenos Aires y decidimos volver. Fue una gran experiencia, no sólo desde el punto de vista profesional sino porque pudimos conocer muy bien recorriendo en auto, los Estados Unidos y Canadá.

Debo decir que la profesión me ha proporcionado muchas oportunidades de viajar y conocer centros importantes. En 1964 fui comisionada por la Universidad de Buenos Aires con el auspicio de la Ford Foundation para asistir a un workshop para estudiosos extranjeros en el Educational Testing Service de Princeton. Allí, que como es sabido es uno de los centros psicométricos más importantes del mundo, tuve oportunidad de estar 2 meses especializándome con el Prof. Wantman y el Prof. Angoff y de conocer a los mejores psicómetras de América Latina. Volqué esta experiencia extraordinaria en mi tercer libro, Manual para la Construcción de Pruebas Objetivas de Rendimiento Escolar publicado por Editorial Paidós en 1968.

En 1967 fui invitada a concurrir como relatora al Paedagogisches Zentrum de Berlin y en 1971 fui invitada por el Dr. Lee Cronbach a concurrir a un congreso de Mental Testing en Estambul (Turquía).

En 1970 volvió el Dr Rimoldi a la Argentina para organizar su centro de investigación, el CIIPME (Centro Interdisciplinario de Investigaciones en Psicología Matemática y Experimental) que dirigió durante muchos años en el CONICET. Yo colaboré invitando a quienes por aquel entonces eran mis ayudantes de cátedra en la UBA y así se conformó el primer equipo del Instituto. Trabajé nuevamente con él como Vicedirectora del CIIPME durante un año en forma totalmente honoraria y me sentí muy feliz de que pudieran comenzar a trabajar con él 8 ó 9 de mis mejores ayudantes de las cátedras de Metodología de la Investigación y de Estadística que ya tenían alguna formación en esta materia y deseaban especializarse en investigación. Ellos conformaron con el Dr. Rimoldi uno de los equipos más 
sólidos del país en investigación psicológica. Actualmente en el CIIPME se sigue trabajando mucho bajo la eficiente dirección de la Dra. Cristina Richaud de Minzi.

Un poco más tarde comenzaron los problemas políticos de todo tipo en la Universidad de Buenos Aires con vaivenes de extrema izquierda y de extrema derecha y yo, como todos, los sufrí. Por suerte frente a algunas incomprensiones encontré a un excelente amigo, el Dr. Horacio Difrieri que me ayudó a pasar los duros años de la dictadura militar, antes de poder jubilarme, refugiada enseñando Estadística en la Carrera de Geografía, período del que guardo también buenos recuerdos.

El resto es casi presente y es difícil escribir objetivamente sobre ello. En 1984 me jubilé y con mi esposo viajamos a los Estados Unidos y a Europa. Pasé unos 6 años alejada de la carrera. En 1992 pensé que tal vez podría hacer algo en investigación y por tercera vez el Dr. Rimoldi tuvo la generosidad de aceptarme para que trabajara en el CIIPME con un contrato del CONICET como Investigador Principal. Allí con un subsidio para un PID realicé una investigación sobre la relación entre logros en educación, inteligencia y resolución de problemas nuevos. Al mismo tiempo en 1994 publiqué mi cuarto libro Diseño Estadístico en el que amplié con varios capítulos sobre técnicas multivariadas y puse al día, mi primer Manual de Estadística, que aún en nuestros días se sigue utilizando como manual de estudio. Finalmente dado que el CONICET no me renovaba el contrato desde 1996 volví nuevamente a la Universidad de Buenos Aires donde el Consejo Directivo de la Facultad de Psicología que dirigía el Decano Dr. Raúl Courel pudo hacerme un contrato como Asesora en Metodología y Estadística para trabajar en el Instituto de Investigación de la Facultad. Además al poco tiempo tuve el honor de que la Universidad de Buenos Aires me diera el Diploma de Profesora Honoraria de la misma. Durante estos últimos años de mi carrera procuro dedicar el mayor tiempo posible a la investigación en forma libre pero siempre doy algunos cursos de postgrado en la Universidad de Buenos Aires y en otras universidades privadas que me suelen contratar.

Comparto muchos proyectos con mi hija Ana, también psicóloga, quien sigue mis pasos. Le gusta mucho la docencia y se entusiasma mucho con hacer investigación. Además siempre tengo ocasión de formar a algunos estudiosos que hacen conmigo su doctorado y concentrarnos en algunos problemas que nos interesan. Por ejemplo, ahora estoy sumamente interesada en los problemas del programa de sesgos y heurísticos iniciado por Tversky y Kahneman, y también estoy profundizando las modernas teorías psicométricas. Así, en el 2004 la Editorial TEA de Madrid me ha publicado un test de aptitud verbal, llamado BAIRES en el que he utilizado la Teoría de la Respuesta al Item. Últimamente he asesorado en Metodología de la Investigación en la Dirección de Orientación al Estudiante. Allí uno de los problemas que más se observa es la poca formación de los alumnos secundarios para seguir estudios universitarios y su falta de información, no solo respecto de las carreras sino hacia el trabajo y las decisiones que han de tomar a través de su trayectoria vital y estamos trabajando en estos problemas que son por otro lado comunes en toda América Latina.

El último gran esfuerzo para mí, ha sido la elaboración del libro Técnicas de Investigación Científica publicado en el año 2008 por Lugar Editorial y que hemos escrito junto con el Dr. López Alonso y y el Dr. Guillermo Macbeth. Este libro me ha hecho reflexionar mucho sobre toda la investigación actual en Psicología y me ha llevado a la conclusión de que es un gran error dividir la investigación psicológica en cualitativa y cuantitativa. La investigación cualitativa tan de moda actualmente, creo que solo debe usarse cuando se comienza un estudio exploratorio para descubrir y entender lo que yace detrás de un fenómeno que se conoce muy poco; pero siempre hay que tener en cuenta que con sus procedimientos nunca se puede constatar una hipótesis y que la investigación es solo una. La verdadera Psicolo- 
gía, si quiere ser ciencia, pienso que debe seguir reforzando sus métodos cuantitativos, como ya se está haciendo, al introducir el análisis de las ecuaciones estructurales, el análisis factorial confirmatorio y otros métodos multivariados, pues pocas veces se puede trabajar con pocas variables. Por otro lado, la gran vía del futuro creo que son los estudios cognitivos neuropsicológicos, en los que en realidad se está muy al comienzo, pero que es un campo sumamente promisorio, sobre todo teniendo en cuenta las nuevas tecnologías para el estudio del cerebro.

En fin, para terminar creo que la Psicología me ha proporcionado una vida muy rica en experiencias. He tenido oportunidad de vivir en varios países diferentes y de viajar a muchos otros. Esto no ha sido obstáculo para que al mismo tiempo construyera con mi esposo, una persona excepcional sin la cual no hubiera podido llegar hasta aquí, una linda familia, con una hija, Ana muy felizmente casada con un excelente compañero, Claudio. Ella también ha querido ser psicóloga, ser docente e investigar, y me ha dado un nieto muy inteligente, llamado Nahuel.

Creo que desde chica tuve gran suerte con los padres y los hermanos que tuve, y posiblemente esto fue lo que me hizo pensar que lo más interesante para conocer en este mundo, era el ser humano. Soy muy sociable y he conocido a toda clase de gente alrededor del mundo y tengo muchos amigos a los que les gustan las mismas cosas que a mí, especialmente los libros y la música clásica.

$\mathrm{Y}$, aunque he tenido como todo el mundo, fracasos y frustraciones, nunca me he arrepentido de haber querido ser psicóloga.

Nuria Cortada de Kohan, Buenos Aires, 8 de ABriL de 2009 